\title{
Comparative Study on Flexible Type of Clutches
}

\author{
Pranav R. Morchapure ${ }^{1}$, Dr K. S. Zakiuddin², Nitin S. Sawarkar ${ }^{3}$ \\ ${ }^{1}$ Research Scholar, Priyadarshini College of Engineering, Nagpur, India,440019 \\ ${ }^{2}$ Professor, Priyadarshini College of Engineering, Nagpur, India, 440019 \\ ${ }^{3}$ Assistant Professor, Priyadarshini College of Engineering, Nagpur, India, 440019
}

DOI: $10.46335 / \mathrm{IJIES} .2020 .5 .9 .7$

\begin{abstract}
In this research, an efficient and reliable analysis technique for the design of flexible clutches has evaluated by using computer modeling. Study mainly focused on stress analysis of the single plate. It is also useful for selecting best-suited clutch for the specific equipment.
\end{abstract}

\section{Keywords: Flexible Clutch, Stress, Modeling}

\section{I- INTRODUCTION}

\section{C}

lutch is a device used in the transmission system of a

vehicle to engage and disengage the transmission system from the engine. Thus, the clutch is located between the engine and the transmission system. In a vehicle, the clutch is always in the engaged position. The clutch is disengaged when starting the engine when shifting gears, when stopping the vehicle and when idling the engine. It disengaged by operating the clutch pedal i.e. by pressing the pedal towards the floor of the vehicle [1]. The clutch engaged when the vehicle has to move and kept in the engaged position when the vehicle is moving. The clutch also permits the gradual taking up of the load, when properly operated; it prevents jerky motion of the vehicle and thus avoids putting undue strain on the remaining parts of the power transmission [2]. It classified into friction clutches and positively Engaged clutch. Friction clutches further classified into cone clutch, centrifugal clutch, and plate clutch. Friction Clutches work based on the frictional forces developed between the two or more surfaces in contact. Friction clutches are usually over the jaw clutches due to their better performance.

\section{II- LITERATURE REVIEW}

Vishal J. Deshbhratar et.al. [1], work contains structural analysis of single plate friction clutch. In his research stress analysis of single plate clutch of the automobile studied. In which the stresses and forces developed in the clutch tried to reduce with the help of software approach .Mamta G. Pawar[2] said clutch system is among the main systems inside a vehicle. Clutch is mechanical device located between a vehicle engine and its transmission and provides mechanical coupling between the engine and transmission input shaft. Clutch system comprises of a flywheel, clutch disc plate and friction material, pressure plate, clutch cover, diaphragm spring and the linkage necessary to operate the clutch. The clutch engages the transmission gradually by allowing a certain amount of slippage between the flywheel and the transmission input shaft.

Namrata Ashok Narkhede et.al. [3],Evaluated modeling of single circular grooves made on dry friction clutches. When there is the working of clutch takes place, there is a huge amount of heat dissipation. Karanjkar A. S. et.al.[4], they have stated in his paper the design of clutch by combining the operation of the centrifugal action in the single plate clutch system of the transmission mechanism to overcome the wearing effect when there is the transmission of power from one shaft to that of the other shaft that is from driving shaft to the driven shaft.

P.Naga Karna et.al.[5], compared the two materials like aluminum and the steel of wet clutch plates by taking the observations of the stress distribution and the temperature distribution of the clutch plate by taking the dimensions of the plate in existence and also take models in the pro-e and the analysis have been taken by using the Ansys. 
Rajesh Purohit, Pooja Khitoliya and Dinesh Kumar Koli [6], Design and finite element analysis of an automotive clutch assembly In the present work a friction clutch assembly was designed. It consists of three parts viz. clutch plate, pressure plate and diaphragm spring. The material assignment is as follows: clutch plate structural steel, pressure plate- cast iron GS-70-02 and diaphragm spring- spring steel. The friction material assumed is moulded asbestos opposing cast iron/ steel surface. Finite element analysis was performed in ANSYS software. The plots for Equivalent Von-Mises stress, total deformation and factor of safety were calculated and analyzed. The finite element analysis showed that the designed friction clutch assembly is safe.

\section{III- PROBLEM IDENTIFICATION}

The problems with a clutch are the material's coefficient of friction, which affected by heat, as shown in fig.1. As the temperature goes up the coefficient of friction goes down, and the total clutch capacity decreases. Heat reduces friction, which increases slippage, heat the cycle becomes self-limiting, when the torque capacity of the clutch drops to zero[6].

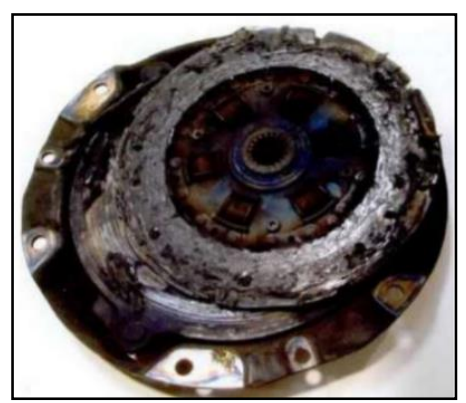

Fig. 1- Friction Material affected by Heat ${ }^{[2]}$

Friction material damaged to or during fit man, overheating due to slip incorrect clutch fitted for the application, shown in fig.2.

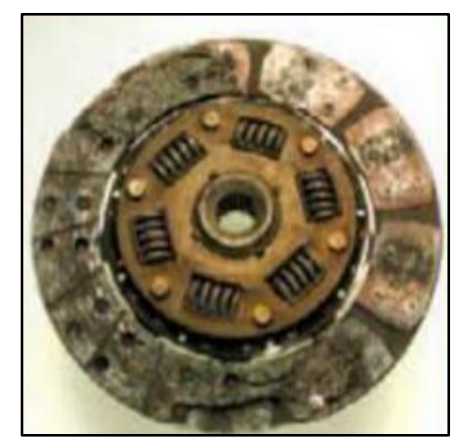

Fig. 2- Fault due to clutch slip ${ }^{[2]}$
On the other side, organic material does have less heat capacity than most other common lining material and it wears fast than some other [7]. The heat created by a Kevlar disc slipping against a flywheel, burns the flywheel. The clutch engages the transmission gradually by allowing a certain amount of slippage between the flywheel and the transmission input shaft. However, the slipping mechanism of the clutch generates heat energy due to friction between the clutch disc and the flywheel. At high sliding velocity, excessive frictional heat is generated which lead to high-temperature rise at the clutch disc surface [8]. This causes thermo-mechanical problems such as thermal deformations and thermoelastic instability, which can lead to thermal cracking, wear and another mode of failure of the clutch disc component.

\section{IV-CONSTRUCTION}

A single plate friction clutch consisting of two flanges shown in fig. 3. One flange rigidly keyed into the driving shaft, while the other is free to move along the driven shaft due to spliced connection.[9] The actuating force is provide by a spring, which forces the driven flange to move towards the driving flange. The face of the drive flange linked with friction material such as cork, leather or Frodo Torque transmitted by plate or disc clutch.

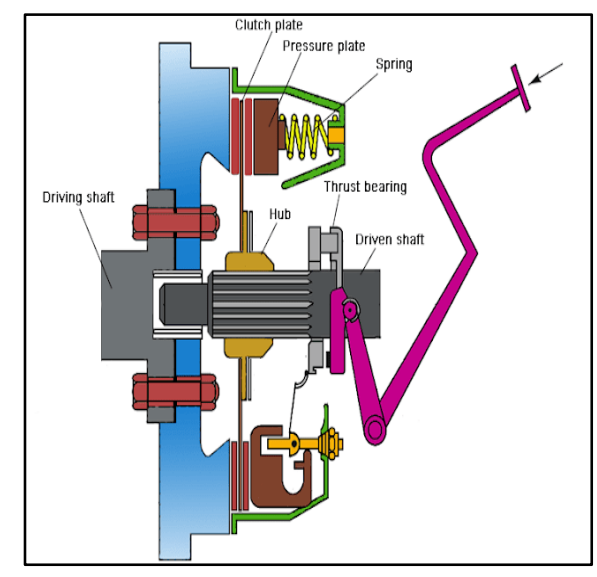

Fig. 3- Single plate clutch

Different parts of single plate clutch arranged in systematical order for its proper laboring. Plate is the most important part of the single plate clutch, which consists of a clutch plate with both side frictionlining.[10]There are some other parts also which helps in the proper functioning of clutch like a flywheel, pressure plate, thrust bearing, hub, springs and 
mechanism for engaging and disengaging of a clutch. The clutch plate moves axially in the driven shaft and attached with the hub between the flywheel and pressure plate.It is must in a single plate clutch for the clutch plate to have both side friction lining because it is mount between the flywheel and pressure plate. In the single-plate clutch, friction is responsible for the torque transmission. The pressure plate is attach with the flywheel and springs. The main function of the pressure plate is to help the clutch plate to move towards the flywheel.[11]A lever is attach to thrust bearings with some mechanism on a driven shaft, which transmits input and output motion from the clutch pedal.

A. Clutch Plate: Clutch Plate is the main component of clutches. There is only one clutch plate present in the single-plate clutch. It is a thin disc-type metallic plate having both side frictional surfaces. These frictional surfaces frictional lining. These frictional lining should be made of such materials that it provides torque transmission without slipping. The coefficient of friction of the materials should be high [12].The clutch plate is assembled between the flywheel and pressure plate.

B. Pressure Plate: The main function of the pressure plate is to maintain proper contact between the surfaces of the flywheel and clutch plate using springs that are attached to it. The pressure plate is generally made of cast iron.

C. Springs: Springs are used to moving the clutch plate towards the flywheel and establish a proper connection between the clutch plate and flywheel. It also prevents the slipping of contact surfaces.

D. Flywheel: The flywheel is connected to the engine output. When the clutch is in an engaged position, the flywheel comes in contact with the clutch plate and torque is transmitted by friction [13].

\section{V- WORKING}

It is the most common type of clutches used in motor vehicles. Single Plate clutch consists of only one clutch plate, mounted on splines of clutch shaft. The flywheel is mounted on engine crankshaft and rotates with it [14]. The pressure plate is bolted on the flywheel with the help of clutch springs and is free to slide when the clutch pedal is pressed. When the clutch pedal is not pressed then the clutch is said to be engaged and at that time the clutch plate is gripped between the flywheel and the pressure plate. There is friction lining on both sides of the clutch plate. When the clutch engaged there is friction between the flywheel, clutch plate and pressure plate. Therefore, as the clutch plate revolves, the clutch shaft also revolves. Clutch shaft connected to the transmission. Thus, the engine power transmitted to the crankshaft to the clutch shaft. When the clutch pedal is pressed, the pressure plate moves back against the force of the springs and the clutch plate becomes free between the flywheel and pressure plates. Hence, the flywheel remains rotating as long as the engine is running and the speed of the clutch shaft reduces slowly and finally, it stops rotating.

\section{VI- STRESSES AND DEFORMATION ON SINGLE PLATE CLUTCH}

Single plate clutch is smooth in operation i.e. engagement and disengagement of the clutch are smooth. Very less slip occurs in its operation. Slip only occurs during the engagement of the clutch and after that no slip. The analysis has made for equivalent stress, total deformation, and heat flux in the friction plate with single circular grooves[14,15,16, and 18]. The study reveals some findings about the behavior of clutch, which is shown in fig.4, 5, \&6. Stresses induced in the plate will be increased as per the use and time interval.

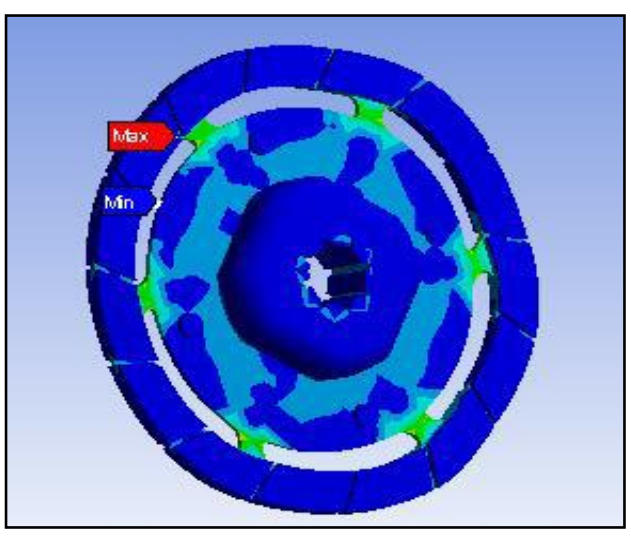

Fig.4- Equivalent Stress ${ }^{[1]}$

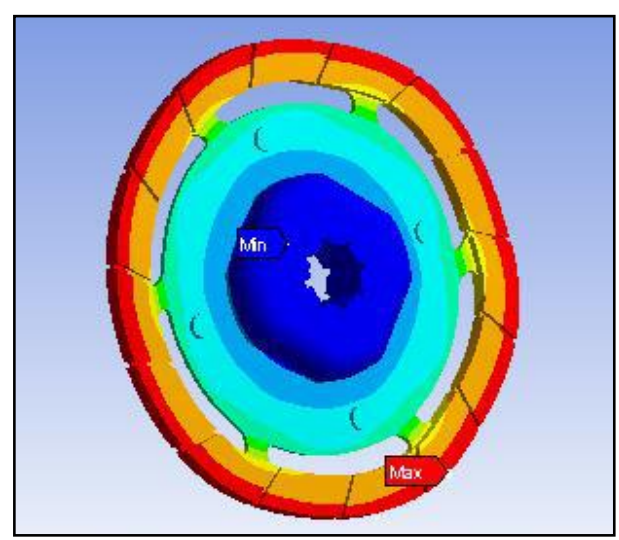

Fig. 5- Total Deformation ${ }^{[1]}$ 


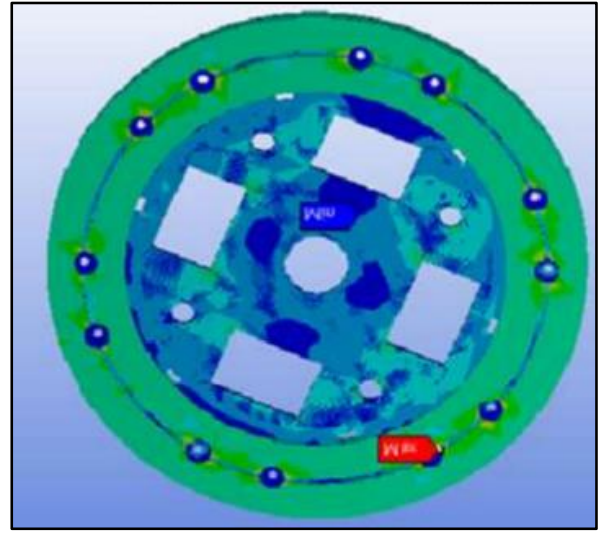

Fig. 6 - Heat Flux in the friction plate with Single Circular grooves $^{[3]}$

While the factors of safety for the three design variants do not vary much, the highest factor of safety is of third design variant. Moreover, as for maximum temperature reached, it is found to be minimum for second design variant while the first design is having very high maximum temperature than the other two designs[7].

Study states that, the capacity of torque transmission is less. The size of this clutch is big even for less torque transmission. It is a clutch so it is required to prevent it from moisture. Chance of tear wear is high in single plate clutches.

\section{VII- CONCLUSION}

After study, it is understood that, the values of equivalent stresses for material loading conditions are less than the allowable stresses for the designs under study. It stated after the stress and thermal analysis in ANSYS.Under such conditions, none of the parts is going to yield and hence, the design is safe.

The results occurred are quite favorable to what were expected. The stress, deformation and temperature clear the idea about what parameter should have been taken into account while defining the single plate friction clutch. The friction clutch is having some obvious demerits like slip, heat between friction parts. The problems with a slipping are that the friction material's coefficient of friction affected by heat. Which cause into the total clutch capacity. Thermo-mechanical problems such as thermal deformations and thermo elastic instability, which can lead to thermal cracking, wear and another mode of failure of the clutch disc component.

\section{REFERENCES}

[1] Design and Structural Analysis of Single Plate Friction ClutchVishal J. Deshbhratar, Nagnath U. Kakde, Vol. 2 Issue 10, October - 2013, pp. 3726-3732

[2] Design and Analysis of Clutch Using Sintered Iron as a Friction Material by Mamta G. Pawar, Monarch K. Warambhe, Gautam R. Jodh,International Journal of Innovative Technology and Exploring Engineering (IJITEE), ISSN: 2278-3075, Volume-3, Issue-7, December 2013

[3] Modelling and Analysis Of Single Circular Grooves Made On Dry Friction Clutches Namrata Ashok Narkhede1, Dheeraj Varma2, IJRET: International Journal of Research in Engineering and Technology, eISSN: 2319-1163 | pISSN: 2321-7308, Volume: 05 Issue: 08 | Aug-2016

[4] Karanjkar A. S., Barve P. C., Adhav R. B., Pandey M. D., Prof. Londhe B. C., Prof. Bhane A. B.[9(ISSN $2347-$ 6435(Online) Volume 4, Issue 4, April 2015)

[5] P. Naga Karna, Tippa Bhimasankara Rao, [11] " eISSN: 2278- 067X, p-ISSN: 2278-800X, Volume 6, Issue 2 (March 2013), PP. $\quad$ 81-87.

[6] Rajesh Purohit, Pooja Khitoliya, Dinesh Kumar Koli, "Design and finite element analysis of an automotive clutch assembly" Procedia Materials Science 6 (2014) $490-502$.

[7] International Journal of Mechanical Engineering and Technology (IJMET) Volume 9, Issue 3, March 2018, pp. 611-618.

[8] Theory of Machines by J. S. Brar, R. K. Bansal 253-255.

[9] May Thin Gyan, Hla Min Htun, Htay Htay Win Ijsetr Design and Structural Analysis ofSingle Plate Clutch" ISSN 2319-8885 Vol.03,(Issue.10)

[10] Structural and Thermal Analysis of a Single Plate Dry Friction Clutch Using FiniteElement Method (Fem) IDL - International Digital Library of Technology \& ResearchVolume 1, (Issue 5), May 2017,

[11] http://www.dbpublications.org/technology\&research/file s/tr_00068.pdf

[12] Analysis of Friction Clutch plate using FEA International Journal of EngineeringResearch and Development e-ISSN: 2278-067X, p-ISSN: 2278-800X, Volume 6, (Issue 2), March 2013, PP. 81-87.

[13] https://en.wikipedia.org/wiki/Clutch

[14] https://www.autodesk.in/solutions/finite-element-analysis

[15] S. Gouse seema begum, A. Balaraju"Design and Analysis of Friction Clutch Plate using Ansys." P.G Student, Department of Mechanical, Madanapalle Institute of Technology \& Science, Madanapalle, Andhra Pradesh, India. Associate Professor, Department of Mechanical, Madanapalle Institute of Technology \& Science, Madanapalle, Andhra Pradesh, India.

[16] Oday I Abdullah ,Josef schlattmann, Michael lytkin "Effect of Surface Roughness on the Thermoelastic Behavior of Friction Clutches. "System Technologies and 
Mechanical Design Methodology, Hamburg University of Technology, Germany.

[17] Talib Ria Jaafar, Mohmad Soib Selamat and Ramlan Kasiran "Selection of Best Formulation for SemiMetallic Brake Friction Materials Development." Talib Ria Jaafarl, Mohmad Soib Selamatl and Ramlan Kasiran2 Advanced Materials Centre, SIRIM Berhad, 34, Jalan Hi-Tech 2/3,Kulim Hi-Tech Park, Kulim.

[18] Shaik Mohammad Ali"Static \& Thermal Analysis of Positive Multiple Friction Plate using FEA Package." $P G$ student, Department of mechanical engineering, Nimra institute of science \& technology, Ibrahimpattanam.

[19] May Thin Gyan, Hla Min Htun, Htay Win "Design and Structural Analysis of Single Plate Clutch." Dept. of Mechanical Engineering, Mandalay Technological University, Mandalay, Myanmar.

[20] Par Nyman, Rikard Maki, Richard Olsson , Bager Ganemi "Influence of surface topography on friction characteristics 